\title{
Online Turkish Handwriting Recognition Using Synthetic Data
}

\author{
Esma Fatıma Bilgin Taşdemir ${ }^{11}$

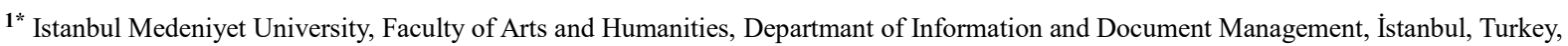 \\ (ORCID: 0000-0002-2465-4186), esmabilgin.tasdemir@medeniyet.edu.tr
}

(International Conference on Design, Research and Development- 15 - 18 Aralık 2021)

(DOI: $10.31590 /$ ejosat.1039846)

\begin{abstract}
ATIF/REFERENCE: Bilgin Taşdemir, E.F., (2021). Online Turkish Handwriting Recognition Using Synthetic Data. European Journal
\end{abstract} of Science and Technology, (32), 649-656.

\begin{abstract}
We present a recognition system for online Turkish handwriting trained with synthetically generated data and transfer learning. Training deep networks requires large amounts of data. However, a sufficiently large collection of Turkish handwriting samples is not available. Hence we synthesize data to do pretraining before adapting the system to target dataset by fine tuning. We generate words from isolated character collection of a large English handwriting dataset. Then, we train the system first with synthetic data and fine tune it with Turkish handwriting samples from a smaller dataset. Fine tuning increases the character recognition rate of the final system which is evaluated on 2,041 samples of isolated Turkish words from the initial value of $61 \%$ to $88 \%$. Performance of the system on synthetic data is quite similar to that on the Turkish test data which shows that the synthetic data resembles the real data quite closely. According to these results, synthetic data generation can be a solution to the data scarcity problem of online Turkish handwriting.
\end{abstract}

Keywords: Turkish Handwriting Recognition, Online Handwriting Recognition, Deep Learning, Synthetic Data Generation, Transfer Learning.

\section{Sentetik Veri Kullanarak Türkçe Çevrimiçi Eı Yazısı Tanıma}

$\ddot{O} \mathbf{z}$

Bu çalışmada yapay veri ve öğrenme tranferi kullanan bir çevrimiçi Türkçe el yazısı tanıma sistemi sunuyoruz. Derin yapay sinir ağlarını eğitmek için çok miktarda veri gerekir. Ancak Türkçe çevrimiçi el yazısı için böylesine büyük bir veri seti bulunmamaktadır. Bu sorunu aşmak için yapay veri üreterek sistemi ön eğitime tabi tutmayı, ardından gerçek veri ile ince ayar yapmayı öneriyoruz. Büyük bir İngilizce çevrimiçi el yazısı veri setindeki ayrık karakter örneklerini kullanarak çevrimiçi el yazısı kelimeler üretiyoruz. Bu yapay veri ile ön eğitime tabi tuttuğumuz sistemi gerçek veri ile de eğiterek 2,041 kelimelik gerçek veri üzerinde test ediyoruz. Öğrenme transferi yöntemi sayesinde Türkçe kelimeler için karakter tanıma oranının \%61'den \%88'e yükseldiğini gözlemliyoruz. Yapay test verisinde de buna yakın bir sonuç alınması yapay verinin gerçek veriye yeterince benzediğini gösterir. Alınan sonuçlara dayanarak yapay veri kullanmanın Türkçe çevrim içi el yazısı alanında yaşanan veri yetersizliği problemine bir çözüm olabileceğini söyleyebiliriz.

Anahtar Kelimeler: Türkçe El Yazısı Tanıma, Çevrimiçi El Yazısı Tanıma, Derin Öğrenme, Sentetik Veri, Öğrenme Transferi.

\footnotetext{
${ }^{1}$ Corresponding Author: esmabilgin.tasdemir@medeniyet.edu.tr
} 


\section{Introduction}

Handwriting recognition is a popular research field with two sub-domains as online and offline recognition according to the representation of the handwritten text to be interpreted by computers. Online data is represented with traces of pen movements which are represented as time series of coordinates. In contrast, offline handwriting is a modality where handwritten text is represented as image data.

Online handwriting is a communication modality which becomes a part of daily life with an ever increasing rate thanks to the widespread use of mobile devices like tablet PCs and smartphones. It is generated by digitizing handwritten symbols, words or lines of words which are captured as coordinates in trace of a pen tip moving on a digitizer equipment. Writer identification and verification and keyword retrieval are two tasks related to handwriting recognition.

Automatic recognition of online handwriting has been an active research area starting with the first studies appearing in the 1940s (Plamondon and Srihari, 2000). Initially, much of the research was focused on the recognition of derivations of Latin alphabet, and especially of English, but other scripts started to gain attention in recent years (Al-Helali and Mahmoud,2017; Doermann and Jaeger, 2008; Plamondon and Srihari,2000; Tagougui et al., 2013).

Recognition can be done at the level of isolated characters and symbols, words, lines and even paragraphs (Carbune et al., 2020; Liwicki and Bunke, 2005a; Priya et al., 2016) .Machine learning techniques like Hidden Markov Models (HMM), Support Vector Machines (SVM) and Artificial Neural Networks (ANN) along with their combinations in hybrid models are heavily used in online handwritten text recognition systems in the literature (Caillault and Viard-Gaudin, 2007; Do and Arti 'eres, 2009; Garcia-Salicetti et al., 2001; Gauthier et al., 2001; Jager et al., 2001; Marukatat et al., 2001; Schenk and Rigoll, 2006; Schenkel et al., 1995). HMM based recognizers was very popular due to their capability of modelling time series effectively, which enabled them to recognize input without segmentation into constituent symbols prior to recognition (Biem, 2006; Hu et al., 2000; Liwicki and Bunke, 2005b; Liwicki et al., 2007a). Like in many other areas, system performances are increased dramatically with the application of Deep Learning (DL) methods in online handwriting recognition domain as well (Graves et al., 2007; Graves et al., 2009; Liwicki et al., 2007a). Particularly, Recurrent Neural Networks (RNNs) and their variants are successfully used for sequential data like online handwriting or speech where data is represented as time series (Husken and Stagge, 2003; Robinson et al., 1996). Long short-term memory neural networks (LSTMs) which solved the so-called "vanishing gradient problem" of RNNs improved the capability of modeling temporal dependencies in data. LSTMs and their variants have been very successful in both online and offline handwritten and machine print text recognition problems in recent years (Carbune et al., 2020; Graves et al., 2007; Graves et al., 2009).

Research about recognition of handwritten Turkish text is very limited. There are studies on offline Turkish character recognition with some constraints applied on the style or the case of writing (Çapar et al., 2003; Kaplan et al., 2017; Korkmaz et al., 2003). In (Yanikoglu and Kholmatov, 2003), a HMM system which was previously developed for English, is used for offline handwritten Turkish text recognition. Some Characters with dots, cedilla and breve which are specific to Turkish alphabet are mapped to their English counterparts. A Turkish prefix parser to detect non-Turkish word prefixes during decoding is employed instead of using a lexicon. A $56 \%$ top-10 word recognition rate is reported using a 17,000-word lexicon. Recognition accuracy decreases to around $40 \%$ when the Turkish prefix parser is used. (Şekerci, 2007) uses HMMs in a character-based word recognition system for offline lowercase mixed-style handwritten Turkish words. The reported recognition rate is $84 \%$ using a lexicon of size 2,500. (Korkmaz et al., 2003), proposes a machine printed character recognizer developed using ANNs. The character recognition rate is reported as $95.2 \%$ for a proprietary dataset. Another character recognizer for offline Turkish handwriting is proposed in (Çapar et al., 2003). Using a classifier based on Size-Dependent Negative Log-Likelihood, a recognition rate of $93.4 \%$ is achieved on a test set of 6,322 samples. A Convolutional Neural Network $(\mathrm{CNN})$ based recognizer is proposed for Turkish handwritten character recognition in (Kabakus and Erdogmus, 2021). Its recognition accuracy is reported as $96.07 \%$.

(Vural et al., 2005) presents a comprehensive evaluation of various HMM architectures and parameters for online handwriting recognition tasks. A word recognition rate of $94 \%$ is achieved using a 1,000-word lexicon with character HMMs. Another HMM system is proposed in (Tasdemir and Yanikoglu, 2018) where data scarcity problem is overcome with using a larger English dataset along with a Turkish dataset containing words taken from elementary school textbooks. $91.7 \%$ word recognition accuracy is reported for a middle-sized, 1,950-word lexicon task. When the lexicon size is increased to 12,500, recognition accuracy is measured as $67.9 \%$, using a bi-gram language model based on word stems and suffixes.

As already mentioned above, DL approaches require availability of plenty of data. It is relatively easier to collect labelled data in the online handwriting domain compared to other research areas like image processing. TabletPCs, smart phones, writing pads and other digitizer equipments are widely available to common users. Yet, the size of publicly available datasets are still limited or sometimes insufficient. For example, IAM-On dataset of English handwriting has 86,272 words in the form of 13,049 lines whereas the ADAB dataset of Arabic handwritten words has 29,922 samples (Boubaker et. al., 2020; Liwicki and Bunke, 2005b). Although they are sufficient to develop experimental systems, these datasets may fall short for representing the real life data perfectly since the latter contains much more variance (Carbune et al., 2020).

Turkish is a special case where data scarcity is quite severe for the online handwriting domain. There are some limited research on recognition of online Turkish handwriting in the literature but the only publicly available dataset is the Elementary Turkish dataset by (Tasdemir and Yanikoglu, 2018) which contains around 10,000 isolated word samples.

In case of data scarcity problem, a popular method is use of synthetic data where large number of labelled data is generated synthetically. Data synthesizing is a widely used technique for the online handwriting domain (Elarian et al., 2014; Lin and Wan, 2007). One of the approaches for generating online handwriting is first modelling the handwriting and then generating samples from the model. Motor model based synthesis techniques (Guerfali and Plamondon, 1995; Plamondon, 1995), stroke and layout modelling (Jawahar et al., 2009) and models using 
modulation of oscillatory motions of the pen (Singer and Tishby, 1994) are examples of that fashion. (Graves, 2013) uses a modelling technique to predict the sampling points generating a handwritten text from real samples. Extracting strokes from offline data and synthesizing online data is another method used in (Haines et al., 2016; Mayr et al., 2020). Another approach is to use units and sub-units like glyphs, characters and strokes of real handwritten texts to generate new data (Ballard et al., 2007; Lin and Wan, 2007; Romero et al., 2012). We take a simple approach similar to (Guyon et al., 1994) and (Romero et al., 2012) where isolated characters written by a particular writer are concatenated to create a word sample.

In this work we come up with a solution to the data scarcity problem in online Turkish handwriting recognition area. We propose to use data synthesized from a handwritten isolated character dataset and use it for training a Convolutional Neural Network-Bidirectional Long-Short Term Memory (CNNBLSTM) network. Further, we do the training in TL fashion to improve the recognition performance of the system. We present results from evaluation of our system as well.

\section{Material and Method}

\subsection{Datasets}

Modern recognition systems require a large number of handwriting samples for training. In general, the larger a dataset, the better it represents the variations in style, slant, skew and contextual differences of character shapes in handwriting. There are some standard datasets that are widely used in online handwriting recognition tasks. In this work we are using two such datasets; UNIPEN for generating synthetic data and ET for applying transfer learning after training with synthetic data.

\subsubsection{UNIPEN}

The UNIPEN online handwriting database is a collection of handwritten samples of digits, characters, words and lines of words (Guyon et al., 1994). The data is created by 40 companies and institutions from all around the world. A total of 5 million characters are created by writers using various writing styles. Also, technical differences in the digitizer equipment of different institutions brings additional variation. The UNIPEN data is considered to be difficult from the perspective of recognition. In this work we use the isolated lower and uppercase English characters collection (category $1 \mathrm{~b}$ and 1c) of the current publicly available version called the train_r01-v07 training dataset. This collection contains 61,351 lowercase and 28,069 uppercase characters, all with various writing styles including handprint and cursive. We use UNIPEN data to generate a collection of words synthetically.

\subsubsection{Elementary Turkish}

Elementary Turkish (ET) dataset is a collection of around 10,000 isolated words written by 113 writers, including children (Tasdemir and Yanikoglu, 2018). Words are selected from a 2,089-word lexicon derived from 1st and 2nd Grade Turkish textbooks. It is split into three sets where writers are not overlapping. The train set contains 7,360 samples from a 1956word lexicon by 79 writers whereas the test set contains 2,500 samples from a 2089-word lexicon written by 34 writers. The train set lexicon covers the test set lexicon. Samples are represented with coordinate values and pen status as up or down of the points extracted from pen traces. We use ET for further training our recognition once it is pre-trained with the synthetic data, and to evaluate it.

\subsubsection{The BOUN Treebank}

The BOUN Treebank is a collection of 9,761 sentences which are sampled from the Turkish National Corpus (TNC) (Turk et al., 2020). TNC is a massive text corpus containing 50 million words from various text types (Aksan et al., 2012). It contains texts from different domains including books, periodicals, social sciences, arts and world affairs. Being a subset of TNC, The BOUN Treebank domains are listed as essays, broadsheet national newspapers, instructional texts, popular culture articles, and biographical texts. A total of 9,761 sentences are randomly selected from these domains. The number of tokens is 121,214. We use words from the BOUN Treebank in generating synthetic data.

\subsection{Recognition System}

We propose a CNN-BLSTM network for recognition of online Turkish handwriting. CNN is a popular DL technique used for feature extraction and different recognition tasks (Li et.al, 2021). RNNs and their variants are proved to be successful in sequence labeling tasks in various domains (Graves, 2012; Shewalkar et al., 2019; Ul-Hasan et al., 2013; Voigtlaender et al., 2016). Hybrid systems where a $\mathrm{CNN}$ is used for feature extraction and a RNN or one of its variants is used as a classifier are widely used for sequence learning tasks (Dutta et al., 2018; Naz et al., 2017; Shi et al., 2017). We take the same approach and use a CNN-BLSTM network for recognition of the online Turkish handwriting task.

The system contains two networks connected in tandem fashion. A CNN network extracts features from the raw data which is made of: $x$ - and $y$-coordinates of the pen movements, differences between the $\mathrm{x}$-and $\mathrm{y}$-coordinates of the current point and the next point and pen status as up or down. Extracted features are fed to a BLSTM network which classifies the features and recognizes the text. 


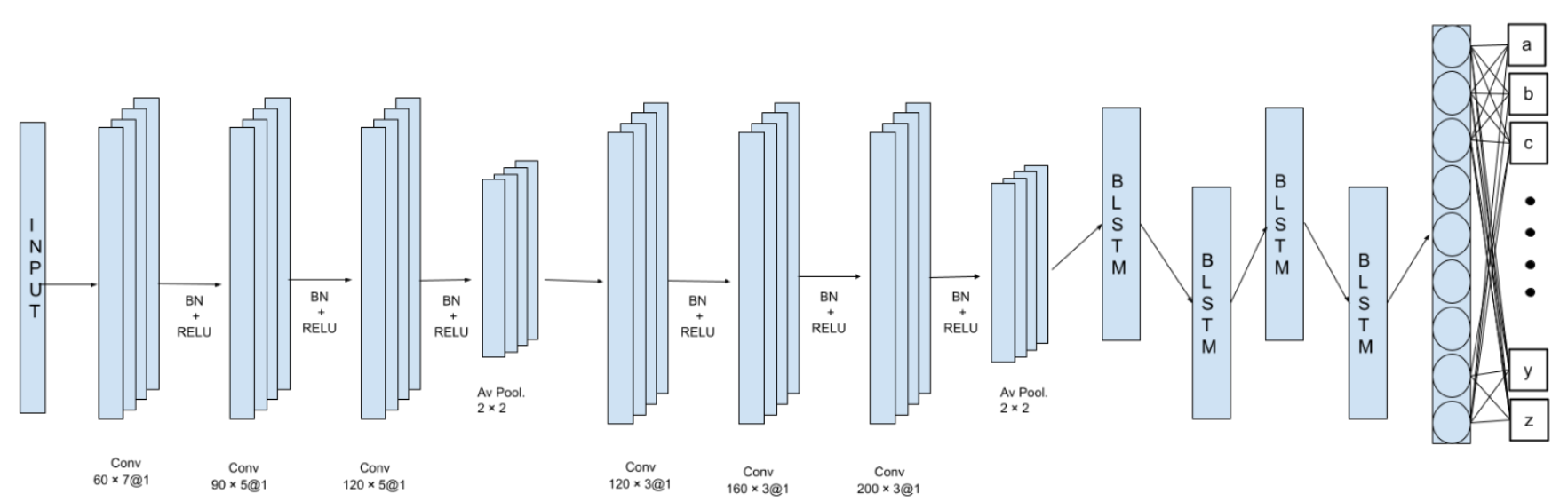

Figure 1. The proposed network architecture.

The CNN network is made of two blocks containing three Convolutional layers each. Batch Normalization is applied after each Convolutional layer. After each block comes an average pooling layer to down sample data representation using a window size of 2. The classifier network has four BLSTM networks stacked together. Each BLSTM layer has two LSTM layers for two directions of processing the feature sequence; as forward and backward. Concatenated output from the LSTM layers are mapped to symbols from the recognition alphabet by means of a softmax layer. The softmax layer calculates the probability of each symbol and assigns chunks of the output from BLSTM to symbols with the highest probability. There are 52 unique symbols used in the UNIPEN samples. The ET dataset contains Turkish letters, upper and lower case and two more symbols. We merge the two symbol sets to obtain a recognition alphabet of 66 symbols. So, the size of the dense softmax layer is 66. CTC loss is calculated using predicted labels from the softmax layer and the ground truth labels. Activation function is ReLU for all trainable layers of the system. Kernels of layers are started using the $\mathrm{He}$ uniform variance scaling initializer. The proposed system architecture is shown in Figure 1.

\subsection{Methodology}

\subsubsection{Synthetic Data Generation}

Training a deep network requires a large dataset with a large number of samples. The only publicly available Turkish online handwriting set is the ET dataset, to the best of our knowledge. There are around 10,000 isolated words in the ET dataset, which is not sufficient to train a DL network from the start. We propose using synthetic data to overcome that problem.

Not every UNIPEN $1 \mathrm{~b}$ and $1 \mathrm{c}$ writer has samples of every character. Also, some writers created multiple samples for the same character. We start with making character sets for each writer. So that characters of the same writer can be used for writing a word which will result in more realistic synthetic data. We use the BOUN Treebank corpus to collect words to be used in synthetic data generation. There are 121,214 tokens in 9,761 sentences in that corpus. Since we want to generate isolated Turkish words using the UNIPEN data, we eliminate tokens which contain symbols other than letters, both upper and lowercase. Finally, we replace Turkish characters \{ç, Ç, ğ $\breve{G}, 1, \dot{\mathrm{I}}$, ö, Ö, ş, Ş, ü, $\ddot{U}\}$ with their counterparts in the English alphabet, i.e. $\{c, C, g, G, i, I, o, O, s, S, u, U\}$ since they are not contained by the UNIPEN dataset. We end up with a word list of 99,479 words and 34,062 unique words. We generate handwritings from that word list by randomly choosing a writer who has samples for all the characters in that word. If there are multiple samples of one character to be written then one of them is chosen randomly. Finally, all chosen character samples are placed on a baseline with randomly chosen spaces between them. The synthetic dataset has 74,859 word samples from a lexicon of 25,223 by 526 writers. Figure 2 shows some examples of the generated words. It should be noted that some of the words in the BOUN Treebank corpus are not included because there is not any writer who has samples for all the characters required to write them.

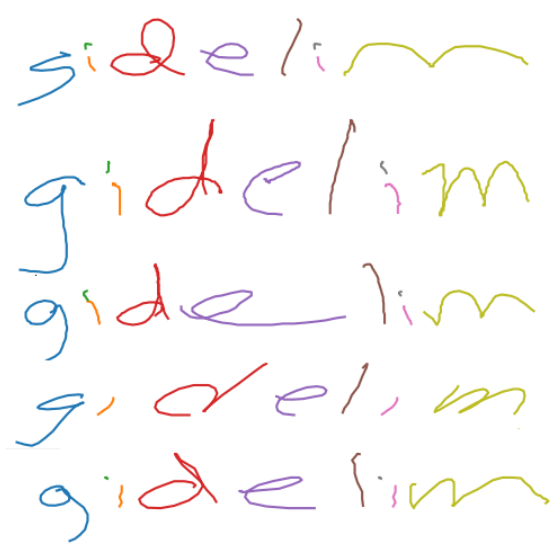

Figure 2. Examples from generated synthetic words.

\subsubsection{System Training}

Although the synthetic data contains samples of Turkish words, they are written with characters coming from a diverse set of writers with different nationalities. Additionally, it lacks some of the Turkish characters which are replaced with their English counterparts. Due to these two issues, we employ the transfer learning approach and make use of the synthetic data. 


\begin{tabular}{l|l|l|l|c|c|c}
\hline Training Set & $\begin{array}{l}\text { Fine tuning } \\
\text { set }\end{array}$ & Testing Set & CER & WER & $\begin{array}{c}\text { Character recognition } \\
\text { accuracy \% }\end{array}$ & $\begin{array}{c}\text { Word recognition } \\
\text { accuracy \% }\end{array}$ \\
\hline Synthetic Train & - & Synthetic Test & 0.006 & 0.024 & 99 & 97 \\
\hline Synthetic Train & - & ET Test & 0.39 & 0.88 & 61 & 12 \\
\hline Synthetic Train & ET Train & Synthetic Test & 0.13 & 0.45 & 87 & 55 \\
\hline Synthetic Train & ET Train & ET Test & 0.12 & 0.44 & 88 & 56 \\
\hline
\end{tabular}

Transfer learning (TL) is a technique for exploiting information obtained during a learning process to improve another learner from a different but related domain. TL is preferred especially in cases where training data is limited. TL has been in use for various problems like text sentiment classification, image classification, and text recognition (Niu et al., 2020; Weiss et al., 2016). Once a DL network is trained with data from a related domain, TL can be applied by freezing some of the layers of which knowledge is accumulated as weights. Freezing prevents the update of the weights. Other layers which will continue learning can be reset or keep learned weights before the network is trained with new data from the target domain. Another method is to fine tune the system with the new data. Here, weights of all layers are updated according to the information learned from the new data. When the domains are similar, fine tuning becomes a more preferable method. According to TL principles, we train our network in two steps; i) first we pre-train the system with the larger synthetic data ii) fine tune it using the ET dataset.

\section{Results and Discussion}

\subsection{Experimental Setup}

Samples of both the UNIPEN and ET datasets are represented by a collection of triples of $\mathrm{x}$-coordinate, $\mathrm{y}$-coordinate and pen up/down status. Strokes are deduced from pen status which always takes the 'up' value except for the last point of each stroke, where it takes the 'down' value. We apply some basic preprocessing to eliminate variations in data. We correct the skew by rotating according to the baseline angle which is calculated by a linear regression. Points are normalized by subtracting mean coordinates values and division by standard deviation of $\mathrm{y}$ coordinates. We resample strokes to obtain equidistant points and finally we up-sample strokes which are shorter than a threshold. After the preprocessing step we use the following elements to represent each point in a stroke:

- its $\mathrm{x}$ - and $\mathrm{y}$-coordinates,

- its difference from the $x$ - and $y$-coordinates of the next point

- its pen status.

Then we split synthetic data samples randomly into three subsets for the pre-training phase. The number of samples in train, validation and test sets are 70,396, 2,000 and 2,000 respectively. In the fine-tuning process, we use approximately $65 \%$ of the data of the ET dataset for training, $10 \%$ for validation during training. $25 \%$ of the samples for evaluating the final system. .Performance evaluation metrics of the proposed system are Character Error Rate (CER) and Word Error Rate (WER) percentages that are based on the Edit Distance. Edit distance is calculated as the minimum number of edits with substitution, insertion and deletion of characters from the reference string to the output, normalized by the number of reference characters. WER is computed in a similar way.

The network is implemented using the TensorFlow library whereas the experiments are run on a NVIDIA GeForce RTX2060 graphical processing unit (GPU) card with 6 GB GPU RAM. The network is trained to minimize the CTC loss function. We use the RMSprop optimization algorithm with an initial learning rate of 0.001 during pre-training and 0.0003 during fine tuning with mini batches of size 20. Training is stopped when the loss on the validation set does not improve after 20 consecutive epochs.

\subsection{Experiments}

We run a series of experiments results of which are shown in Table 1. The system is first trained with isolated synthetic Turkish word samples. Its character recognition accuracy and CER are measured as $99 \%$ and 0.006 respectively on test set of the synthetic data. The same system obtains $61 \%$ accuracy at character level and 0.39 CER and on the test set of the ET dataset. The system is trained further with ET train data to apply TL in the form of fine tuning. The initial learning rate which was 0.001 during the pre-training phase is lowered to 0.0003 for the fine tuning step. After validation stop occurs, we test the fine-tuned system and measure character recognition accuracy as $88 \%$ and CER as 0.12 on the ET test set.

\subsection{Discussion and Error Analysis}

According to the results, the synthetic word dataset is useful for training a general handwriting recognition system. A huge increase is observed in recognition accuracy for the ET data after TL training. We can say that the real data brings a huge improvement over the general recognition system by fine tuning the system. On the other hand, recognition accuracy for the synthetic data drops by 12 points after applying TL which is an expected result. Actually, recognition accuracy rates and CER are almost the same for synthetic data and ET data which shows the synthetic data resembles the real data quite closely.

Much of the errors are due to the confusion between characters written with similar shapes. In most cases, only one of the characters in a word is misrecognized. Applying a spell checker in case of such errors can easily produce the correct 
words. Indeed, if we omit the case errors, word recognition accuracy increases to $62 \%$. We do not observe any particular difficulty for Turkish letters that are not included in the English alphabet. Table 2 shows examples of recognition errors.

Our results are not directly comparable to results from the literature which are quite small in number. (Vural et al., 2005) reports a word recognition rate of $94 \%$ achieved using a 1,000 word lexicon with character HMMs. They use proprietary data which is not available to the public.

Table 2. Examples from recognition errors.

\begin{tabular}{|c|c|c|c|}
\hline $\begin{array}{l}\text { Ground } \\
\text { Truth }\end{array}$ & Predicted & $\begin{array}{l}\text { Ground } \\
\text { Truth }\end{array}$ & Predicted \\
\hline korkuyor & korkuyo & çekti & cekli \\
\hline$U Y U D U \breve{G} U$ & uyuduğu & ağırdı & agırdl \\
\hline horozu & horozl & üzerini & üicerini \\
\hline Etkinlik & etkinlik & ettim & ettım \\
\hline geçtiği & gectiği & çarpıyordu & çarpıyardı \\
\hline bölüp & bölüşs & Ayrıca & Ayırıca \\
\hline boğulacă̆ımı & boğulacă̆ını & şeklin & seklin \\
\hline girdiğinde & girdiğinede & kaldırıp & kaldırip \\
\hline gösterdi & Gösterdi & Görsellerin & Giörsellerin \\
\hline diğer & diğet & gidiyorduk & gidiyarduk \\
\hline yaşarız & yalarız & cekli & çekti \\
\hline paragrafa & Pară̆rafa & agırd $\iota$ & ağglrdı \\
\hline yorulmuş & yoruluuş & üicerini & üzerini \\
\hline seklin & şeklin & ettım & ettim \\
\hline kaldırip & kaldırıp & çarpıyardı & çarpıyordu \\
\hline
\end{tabular}

In (Tasdemir and Yanikoglu, 2018), 91.7\% word recognition accuracy is reported for a 1,950-word lexicon task. When the lexicon size is increased to 12,500 , recognition accuracy is measured as $67.9 \%$, using a bi-gram language model based on word stems and suffixes. Although the latter uses the ET dataset, our work is not directly comparable to that last one since it is a closed-dictionary task (lexicon size 1,956 and 12,500). Our system is tested in an open-dictionary task. Furthermore they use a language model whereas we do not employ one. According to these results, we can say that performance of the proposed system is on par with the results from the previous studies.

\section{Conclusions and Recommendations}

This work proposes to use synthetic data to develop an online handwriting recognition system for Turkish. The system achieves state-of-art results comparable to those published in the literature. Using isolated character datasets of the large UNIPEN collection, we generate a synthetic online Turkish isolated words dataset. Then we train a CNN-BLSTM network using the synthetic data. Later we apply transfer learning by fine tuning the already trained network using the real Turkish handwriting samples. Fine tuning increases recognition accuracy of the final system on 2,041 Turkish samples from $61 \%$ to $88 \%$. We conclude that using the synthetic data and the TL technique together can overcome the data scarcity problem in the Turkish handwriting recognition domain.

Using a language model, training with an even larger corpus, using character samples from a larger number of writers in data generation and post-processing the results can improve the system performance dramatically.

\section{References}

Aksan, Y., Aksan, M., Koltuksuz, A., Sezer, T., Mersinli, Ü., Demirhan, U. U., Yilmazer, H., Atasoy, G., Öz, S., Yildiz, I. \& Kurtoglu, Ö. (2012). Construction of the Turkish National Corpus (TNC). In N. Calzolari, K.

Choukri, T. Declerck, M. U. Dogan, B. Maegaard, J. Mariani, J. Odijk \& S. Piperidis (Hrsg.), Proceedings of the Eighth International Conference on Language Resources and Evaluation, LREC2012, Istanbul, Turkey, May23-25,2012 (S. 3223-3227). European Language Resources Association (ELRA).

Al-Helali, B. M. \& Mahmoud, S. A. (2017). Arabic Online Handwriting Recognition (AOHR): A Survey. ACM Comput. Surv., 50(3), 33:1-33:35.

Ballard, L., Lopresti, D. P. \& Monrose, F. (2007). Forgery Quality and Its Implications for Behavioral Biometric Security. IEEE Trans. Syst. Man Cybern. Part B, 37(5), 1107-1118.

Biem, A. (2006). Minimum classification error training for online handwriting Recognition. IEEE Trans. Pattern Anal. Mach. Intell., 28(7), 1041-1051.

Caillault, É. \& Viard-Gaudin, C. (2007). Mixed Discriminant Training of Hybrid ANN/HMM Systems for Online Handwritten Word Recognition. IJPRAI, 21(1), 117-134.

Boubaker, H. , Elbaati, A., Tagougui, N., El Abed, H., Kherallah, M., Märgner, V., \& Alimi, A. M. (2021). ADAB database. IEEE Dataport.

Li, Z., Liu, F., Yang, W., Peng, S. \& Zhou, J. "A Survey of Convolutional Neural Networks: Analysis, Applications, and Prospects," in IEEE Transactions on Neural Networks and Learning Systems,

Çapar, A., Tasdemir, K., Kilic, Ö. \& Gökmen, M. (2003). A Turkish Handprint Character Recognition System. Computer and Information Sciences - ISCIS 2003, 18th International Symposium, Antalya, Turkey, November 3-5, 2003, Proceedings, 447-456.

Carbune, V., Gonnet, P., Deselaers, T., Rowley, H. A., Daryin, A. N., Calvo, M., Wang, L., Keysers, D., Feuz, S. \& Gervais, P. (2020). Fast multi-language LSTM-based online handwriting recognition. Int. J. Document Anal. Recognit., 23(2), 89-102.

Do, T. M. T. \& Artières, T. (2009). Maximum Margin Training of Gaussian HMMs for Handwriting Recognition. 10th International Conference on Document Analysis and Recognition, ICDAR2009, Barcelona, Spain, 26-29 July 2009, 976-980.

Doermann, D. S. \& Jaeger, S. (Hrsg.). (2008). Arabic and Chinese Handwriting Recognition SACH2006 Summit College Park, 
MD, USA, September 27-28, 2006 Selected Papers (Bd. 4768). Springer.

Dutta, K., Krishnan, P., Mathew, M. \& Jawahar, C. V. (2018). Improving CNN-RNN Hybrid Networks for Handwriting Recognition. 16th International Conference on Frontiers in Handwriting Recognition, ICFHR 2018, Niagara Falls, NY, USA, August 5-8, 2018, 80-85.

Elarian, Y., Abdel-Aal, R. E., Ahmad, I., Parvez, M. T. \& Zidouri, A. B. C. (2014). Handwriting synthesis: classifications and techniques. Int. J. Document Anal. Recognit., 17(4), 455-469.

Garcia-Salicetti, S., Dorizzi, B., Gallinari, P. \& Wimmer, Z. (2001). Maximum Mutual Information training for an online neural predictive handwritten word recognition system. IJDAR, 4(1), 56-68.

Gauthier, N., Artières, T., Gallinari, P. \& Dorizzi, B. (2001). Strategies for Combining On-line and Off-line Information in an On-line Handwriting Recognition System. 6th International Conference on Document Analysis and Recognition, ICDAR 2001, 10-13 September 2001, Seattle, WA, USA, 412-416.

Graves, A. (2012). Supervised Sequence Labelling with Recurrent Neural Networks (Bd. 385). Springer.

Graves, A. (2013). Generating Sequences With Recurrent Neural Networks. CoRR, abs/1308.0850.

Graves, A., Fernández, S., Liwicki, M., Bunke, H. \& Schmidhuber, J. (2007). Unconstrained on-line handwriting recognition with recurrent neural networks. Advances in Neural Information Processing Systems, Proceedings of the Twenty-First Annual Conference on Neural Information Processing Systems, NIPS, Vancouver, British Columbia, Canada, December 3-6, 2007, 577-584.

Graves, A., Liwicki, M., Fernandez, S., Bertolami, R., Bunke, H. \& Schmidhuber, J. (2009). A novel coNNectionist system for unconstrained handwriting recognition. IEEE Trans. Pattern Anal. Mach. Intell., 31(5), 855-868.

Guerfali, W. \& Plamondon, R. (1995). The Delta LogNormal theory for the generation and modeling of cursive characters. Third International Conference on Document Analysis and Recognition, ICDAR 1995, August 14 - 15, 1995, Montreal, Canada. Volume I, 495-498.

Guyon, I., Schomaker, L., Plamondon, R., Liberman, M. \& Janet, S. (1994). UNIPEN project of on-line data exchange and recognizer benchmarks. 12th IAPR International Conference on Pattern Recognition, Conference B: Patern Recognition and Neural Networks, ICPR 1994, Jerusalem, Israel, 9-13 October, 1994, Volume 2, 29-33.

Haines, T. S. F., Aodha, O. M. \& Brostow, G. J. (2016). My Text in Your Handwriting. ACM Trans. Graph., 35(3), 26:1-26:18.

Hu, J., Lim, S. G. \& Brown, M. K. (2000). Writer independent online handwriting recognition using an HMM approach. Pattern Recognition, 33(1), 133-147.

Hüsken, M. \& Stagge, P. (2003). Recurrent neural networks for time series classification. Neurocomputing, 50, 223-235.

Jäger, S., Manke, S., Reichert, J. \& Waibel, A. (2001). Online handwriting recognition: the NPen++ recognizer. IJDAR, 3(3), 169-180.

Jawahar, C. V., Balasubramanian, A., Meshesha, M. \& Namboodiri, A. M. (2009). Retrieval of online handwriting by synthesis and matching. Pattern Recognit., 42(7), 14451457.

Kabakus, A. T. \& Erdogmus, P. (2021). A novel handwritten Turkish letter recognition model based on convolutional neural network. Concurr. Comput. Pract. Exp., 33(21).
Kaplan, K., Ertunç, H. M. \& Vardar, E. (2017). Handwriting Character Recognision by using Fuzzy Logic. Firat University Turkish Journal of Science \& Technology, 12, 7177.

Korkmaz, S. U., Kirçiçegi, G., Akinci, Y. \& Atalay, V. (2003). A Character Recognizer for Turkish Language. 7th International Conference on Document Analysis and Recognition (ICDAR 2003), 2-Volume Set, 3-6 August 2003, Edinburgh, Scotland, UK, 1238-1241.

Lin, Z. \& Wan, L. (2007). Style-preserving English handwriting synthesis. PatternRecognit., 40(7), 2097-2109.

Liwicki, M. \& Bunke, H. (2005a). Handwriting recognition of whiteboard notes. In Proceedings of the 12th Conference of the International Graphonomics Society, 118-122.

Liwicki, M. \& Bunke, H. (2005b). IAM-OnDB - an On-Line English Sentence Database Acquired from Handwritten Text on a Whiteboard. Eighth International Conference on Document Analysis and Recognition (ICDAR 2005), 29 August - 1 September 2005, Seoul, Korea, 956-961.

Liwicki, M., Graves, A., Bunke, H. \& Schmidhuber, J. (2007a). A novel approach to on-line handwriting recognition based on bidirectional long short-term memory networks. In Proceedings of the 9th International Conference on Document Analysis and Recognition, ICDAR 2007, 367371.

Marukatat, S., Artières, T., Gallinari, P. \& Dorizzi, B. (2001). Sentence Recognition through Hybrid Neuro-Markovian Modeling. 6th International Conference on Document Analysis and Recognition, ICDAR 2001, 10-13 September 2001, Seattle, WA, USA, 731-737.

Mayr, M., Stumpf, M., Nicolaou, A., Seuret, M., Maier, A. \& Christlein, V. (2020). Spatio-Temporal Handwriting Imitation. In A. Bartoli \& A. Fusiello (Hrsg.), Computer Vision - ECCV 2020 Workshops - Glasgow, UK, August 2328, 2020, Proceedings, Part V (S. 528-543). Springer.

Naz, S., Umar, A. I., Ahmad, R., Siddiqi, I., Ahmed, S. B., Razzak, M. I. \& Shafait, F. (2017). Urdu Nastaliq recognition using convolutional-recursive deep learning. Neurocomputing, 243, $80-87$.

Niu, S., Liu, Y., Wang, J. \& Song, H. (2020). A Decade Survey of Transfer Learning (2010-2020). IEEE Trans. Artif. Intell., 1(2), 151-166.

Plamondon, R. (1995). A kinematic theory of rapid human movements. Biological Cybernetics, 72, 295-307.

Plamondon, R. \& Srihari, S. N. (2000). On-line and off-line handwriting recognition: A Comprehensive Survey. IEEE Trans. Pattern Anal. Mach. Intell., 22(1), 63-84.

Priya, A., Mishra, S., Raj, S., Mandal, S. \& Datta, S. (2016). Online and offline character recognition: A survey. 2016 International Conference on Communication and Signal Processing (ICCSP), 0967-0970.

Robinson, T., Hochberg, M. \& Renals, S. (1996). The Use of Recurrent Neural Networks in Continuous Speech Recognition. In C.-H. Lee, F. K. Soong \& K. K. Paliwal (Hrsg.), Automatic Speech and Speaker Recognition: Advanced Topics (S. 233-258). Springer US.

Romero, V., Rossi, A. H. T. \& Vidal, E. (2012). Multimodal Interactive Handwritten Text Transcription (Bd. 80). WorldScientific.

Schenk, J. \& Rigoll, G. (2006). Novel hybrid NN/HMM modelling techniques for on-line handwriting recognition. 10th International Workshop on Frontiers in Handwriting 
Recognition, IWFHR 2006, IAPR. , La Baule, France, Oct 2006, 619-6230.

Schenkel, M., Guyon, I. \& Henderson, D. (1995). On-line cursive script recognition using Time-delay Neural Networks and Hidden Markov Models. Mach. Vis. Appl., 8(4), 215-223.

Şekerci, M. (2007). Turkish connected and slant handwriting recognition system. ( MSc thesis ). Trakya Üniversitesi.

Shewalkar, A., Nyavanandi, D. \& Ludwig, S. A. (2019). Performance Evaluation of Deep Neural Networks Applied to Speech Recognition: RNN, LSTM and GRU. J. Artif. Intell. Soft Comput. Res., 9(4), 235-245.

Shi, B., Bai, X. \& Yao, C. (2017). An End-to-End Trainable Neural Network for Image-Based Sequence Recognition and Its Application to Scene Text Recognition. IEEE Trans. Pattern Anal. Mach. Intell., 39(11), 2298-2304.

Singer, Y. \& Tishby, N. (1994). Dynamical encoding of cursive handwriting. Biol. Cybern., 71(3), 227-237.

Tagougui, N., Kherallah, M. \& Alimi, A. M. (2013). Online Arabic handwriting recognition: a survey. IJDAR, 16(3), 209-226.

Tasdemir, E. F. B. \& Yanikoglu, B. A. (2018). Large vocabulary recognition for online Turkish handwriting with sublexical units. Turkish J. Electr. Eng. Comput. Sci., 26(5), 2218-2233.

The UNIPEN Consortium. (n. d.). The UNIPEN project [Accessed: 2017-12-26]. http://www.unipen.org/products.html

Türk, U., Atmaca, F., Özates, S. B., Berk, G., Bedir, S. T., Köksal, A., Basaran, B. Ö., Güngör, T. \& Özgür, A. (2020). Resources for Turkish Dependency Parsing: Introducing the BOUN Treebank and the BoAT Annotation Tool. CoRR, abs/2002.10416.

Ul-Hasan, A., Ahmed, S. B., Rashid, S. F., Shafait, F. \& Breuel, T. M. (2013). Offline Printed Urdu Nastaleeq Script Recognition with Bidirectional LSTM Networks. 12th International Conference on Document Analysis and Recognition, 1061-1065.

Voigtlaender, P., Doetsch, P. \& Ney, H. (2016). Handwriting Recognition with Large Multidimensional Long Short-Term Memory Recurrent Neural Networks. 15th International Conference on Frontiers in Handwriting Recognition, 228233.

Vural, E., Erdogan, H., Oflazer, K. \& Yanikoglu, B. A. (2005). An online handwriting recognition system for Turkish. Document Recognition and Retrieval XII, DRR2005, SanJose, California, USA, January 16-20, 2005, Proceedings, $56-65$.

Weiss, K. R., Khoshgoftaar, T. M. \& Wang, D. (2016). A survey of transfer learning. J.BigData, 3, 9.

Yanikoglu, B. \& Kholmatov, A. (2003). Turkish handwritten text recognition: a case of agglutinative languages. Document Recognition and Retrieval X, 22-23 January 2003, Santa Clara, California, USA, Proceedings, 227-233. 